\title{
PREDICTIVE VARIABLES OF PATHOLOGIC COMPLETE RESPONSE (PCR) IN INVASIVE DUCTAL CARCINOMA
}

Iris Rabinovich¹, Isabela Chaves Monteiro Soares', Julia Mizrahi Jakobson¹, Eduardo Milleo Kac¹, Bruno Luiz Zonta 'Universidade Federal do Paraná - Curitiba (PR), Brazil.

Introduction: Achieving a pCR after neoadjuvant chemotherapy is generally associated with better outcomes. Identifying modifiable and non-modifiable variables that correlate with a complete response is important to treatment. Objectives: The aim of this study was to identify clinical and immunohistochemical variables that correlate with pCR, in a public and a private breast cancer unit in the city of Curitiba, state of Paraná, Brazil. Methods: A retrospective analysis was carried out through medical records at Hospital de Clínicas- UFPR and Centro de Doenças da Mama- Hospital Nossa Senhora das Graças. Patients who underwent surgical treatment after neoadjuvant chemotherapy, in the period from January 1, 2017 to December 31, 2020, due to an invasive ductal carcinoma were selected. Clinical and immunohistochemical variables were correlated to the presence of pCR. Ethical committee approval n. 4,295,049. Results: In 240 cases included, 66 (27.5\%) reached pCR. There was a significant correlation between $\mathrm{pCR}$ and the negativity of the hormonal receptors $(\mathrm{p}=0.002)$, HER2 positivity ( $\mathrm{p}=0.002)$, Ki67 expression ( $\mathrm{p}=0.012)$ and molecular subtypes $(\mathrm{p}=0.001)$. A ROC (receiver operating characteristic) curve analysis identified a 50\% percentage as the best cut-off value to predict complete response. On the other hand, it was not observed a significant correlation between $\mathrm{pCR}$ and body mass index, or physical activity. Conclusions: There is a strong correlation between immunohistochemical parameters and pCR. Further studies are needed to determine the correlation between modifiable variables. 